\title{
Cytogenetics and evolution of cassava (Manihot esculenta Crantz) ${ }^{*}$
}

\author{
Nagib M.A. Nassar \\ Departamento de Genética e Morfologia, Universidade de Brasília, 70919 Brasília, DF, Brasil. \\ *Dedicated to Prof. Francisco Duarte, Founder of the Genetics and Molecular Biology on 25 years of successful editorship.
}

\begin{abstract}
All Manihot species so far examined, including cassava (Manihot esculenta), have $2 \mathrm{n}=36$. Interspecific hybrids between cassava and its wild relatives show fair regular meiosis, and backcrossed generations exhibit high fertility. Electrophoresis shows affinity between species of different sections, as well as between some of them and cassava itself. Polyploidy has apparently contributed to the rapid speciation of this genus, while apomixis has offered a means of perpetuating new hybrid types adapted to different environments. It is assumed that cassava originated by hybridization between two wild Manihot species followed by vegetative reproduction of the hybrid.
\end{abstract}

\section{MANIHOTSYSTEMATICS}

Cassava (Manihot esculenta Crantz) does not grow wild. About 98 species are known to belong to the genus Manihot (Rogers and Appan, 1973), ranging from subshrubs to shrubs and trees, the majority of them producing latex and containing cyanogenic glucosides. Unlike the cultivated species, the roots of wild species are fibrous and slender, but some species frequently exhibit a limited number of tuberous roots, the surfaces of which may be smooth or rough with a sub-epidermis varying from red or yellow to white, while the cortex of tuberous rooted species are white, cream or yellow.

Stem height varies from almost acaulescent in subshrubs to about $20 \mathrm{~m}$ in tree species. Shrub-type species native to the Brazilian savanna frequently have their stems die back to the crown in the dry season, while stem color varies from gray to brown or reddish. Stems normally branch in a dichotomous or trichotomous manner, with the branching point exhibiting a terminal inflorescence. In wild species the young stem frequently has a varying degree of pubescence, a character rarely encountered in the cultigen cassava (Grattapaglia et al., 1986).

Leaves are alternate, varying from subsessile to long petiolated and all except 3 species had palmately lobed leaf. Inflorescences are terminal and monoeceous with the exception of acaulescent species native to central Brazil. Flowers have a single perianth composed of 5 petals, with their length ranging from 0.5 to $2.0 \mathrm{~cm}$. The buds of staminate flowers are ovoid or spheric, while those of pistillate flowers are conic. The fruits are capsular with three locules, while the seeds have a caruncle which var- ies in size. The chromosome number in all species investigated is $2 \mathrm{n}=36$ (Nassar, 1978a).

All species of the genus Manihot are native to countries of the New World, especially Brazil and Mexico, where they form distinct centers of diversity (Nassar, 1978b). They normally grow sporadically in their habitat and rarely become the dominant vegetation. Due to the monoeceous or dioecious structure of the inflorescence, wild Manihot species are typically alogamous plants, but in cultivated cassava a shift towards autogamous plants has occurred which Nassar and O'Hair (1985) have explained by the monoclonal system of cultivation and the domestication history of the cultigen. Observations of frequent hybridization between the wild species and the cultigen as well as between the wild species themselves suggest weak inter-species fertilization barriers (Nassar, 1980a), probably due to the polyploid nature of the genus.

\section{DISTRIBUTION OF WILD MANIHOTSPECIES}

During May and July 1975 the author made a trip to three northeastern Brazilian states (Pernambuco (PE), Ceará (CA) and Bahia (BA)) and collected seeds of wild Manihot species. The geographical distribution of $M a-$ nihot species had already been studied by Rogers and Appan (1973), and Manihot specimens collected by the Reading University Expedition (RUE) and deposited at IPA herbarium, Recife, were also examined.

Table I lists the wild species of Manihot that were collected from different localities of northeastern Brazil, and it is apparent that western Pernambuco and central Bahia had the greatest variability in Manihot species. Some species reported by the RUE as occurring in some of these localities could not be found, e.g., specimens of M. glaziovii (which unlike most Manihot species grows in large numbers and not as sporadic plants) collected by the RUE about $12 \mathrm{~km}$ west of the town of Ibimirim, PE, could not be found because the vegetation in that area had been cleared and the land cultivated with the castor-oil plant (mamona in Portuguese). Extinction of some wild Manihot species in their natural habitat may be due to another factor, i.e., the majority of these species are poisonous to grazing animals because of the presence of $\mathrm{HCN}$ and are known among the people of northeastern Brazil as maniçoba (literally "the poisonous cassava"), and because of these many plants are destroyed by farmers. 
Table I - Wild species of Manihot

collected from different localitles in northeastern Brazil.

\begin{tabular}{|ll|}
\hline Species & Locality \\
\hline M. caerulescens Pohl & Aparipina, PE \\
M. heptaphylla Ule & Seabra, BA \\
M. cichotoma Ule & Jequié, BA \\
$\begin{array}{l}\text { M. } \text { catingae } \text { Ule } \\
\text { M. brachyandra } \text { Pax }\end{array}$ & Itaberaba, BA \\
et Hoffmann & Petrolina, PE \\
$\begin{array}{l}\text { M. } \text { maracasensis } \text { Ule } \\
\text { M. } \text { epruinosa } \text { Pax }\end{array}$ & Itambé, BA \\
et Hoffmann & Bentecoste, Fortaleza, CE \\
M glaziovii Mueller & Arcoverde, Ouricure, Serratalada, PE \\
M. jacobinensis Mueller & Vitoria da Conquista, BA \\
M. quinquefolia Pohl & Senhor do Bonfim, Juazeiro, BA \\
\hline
\end{tabular}

By studying the geographical distribution of the Manihot species found by Rogers and Appan (1973) as well as during my 1975 trip it was possible to construct a map of the distribution of wild species. This map shows that central Brazil (southern Goiás and eastern Minas Gerais) has about 38 wild species from a total 98 recognized species, the highest known diversity for any region.

Manihot species occurring in the first diversity center (southern Goiás and eastern Minas Gerais, Brazil)

M. acuminatissima Mueller, $M$. sparsifolia Pohl, $M$. pruinosa Pohl, M. alutacea Rogers \& Appan, M. divergens Pohl, M. cecropiaefolia Pohl, M. triphylia Polh, M. pentaphylla Pohl, M. anomala Pohl, M. procumbens Mueller, M. crotalariaeformis Pohl, M. pusilla Pohl, $M$. Iogepetiolata Pohl, M. tomentosa Pohl, M. purpureocostata Pohl, M. attenuata Mueller, M. orbicularis Pohl, $M$. tripartita (Sprengel) Mueller, M. pilosa Pohl, $M$. sagittato-partita Pohl, M. falcata Rogers \& Appan, $M$. quinqueloba Pohl, M. violacea Pohl, M. irwinii Rogers \& Appan, M. mossamedensis Taubet, M. puticulosa (Pax) Rogers \& Appan, $M$. gracilis Pohl, $M$. warmingii Mueller, $M$. reptans $\mathrm{Pax}, M$. stipularis $\mathrm{Pax}, M$. oligantha $\mathrm{Pax}, M$. nana Mueller, $M$. stricta Baillon, $M$. salicifolia Pohl, $M$. weddelliana Baillon, M. peltata Pohl, M. janiphoides Mueller and M. handroana N. D. Cruz.

Manihot species which occur in the second diversity center (southwestern Mexico)

M. pringlei Watson, M. aesculifolia Pohl, M. oaxaca Rogers \& Appan, M. rhomboidea Mueller, M. walkarae Croizat, M. divisiae Croizat, M. michaelis McVaugh, $M$. websterae Rogers \& Appan, M. auriculata McVaugh, $M$. rubricaulis I.M. Hohnson, M. chlorosticta Standley \& Goldman, M. subspicata Rogers \& Appan, M. caudata Greenman, M. angustiloba (Torrey) Mueller, M. tomatophylla Standley and M. foetida Pohl.
Manihot species which occur in the third diversity center (northeastern Brazil)

M. zenAtneri Ule, M. surinamensis Rogers \& Appan, M. quinquefolia Pohl, M. pseudoglaziovii Pax \& Hoffmann, $M$. maracasensis Ule, $M$. quinquepartita Huber, $M$. caerulescens Pohl, M. marajoara Chermont de Miranda, M. tristis Mueller, M. glaziovii Mueller, M. epruinosa Paz \& Hoffmann, $M$. brachyandra Pax \& Hoffmann, $M$. dichotoma Ule, M. Ieptophylla Pax, M. reniformis Pohl and M. heptaphylla Ule.

\section{Manihot species which occur in the fourth diversity center (southwestern Mato Grosso (Brazil) and Bolivia)}

M. guaranitica Choda \& llassier, M. pruinosa Pohl, M. jacobinsis Mueller, M. condesata Rogers \& Appan, $M$. xavantinensis Rogers \& Appan and M. flemingiana Rogers \& Appan.

Vavilov has shown that variation in cultivated plants is confined to a relatively few restricted areas or centers and that these diversity centers are the places of origin of cultivated plants. In 1920 he suggested that there were six main geographical centers for the origin of cultivated plants, which in 1935 he later increased to about ten (Vavilov, 1951). He assumed that the Brazilian-Bolivian cassava center is the main cassava diversity center. Since the development of the concept of diversity centers in the 1920's, much more information has been gathered and it has become clear that not all diversity centers represent centers of origin. Harlan (1961) has shown that more than one diversity center may be formed for a given crop through introgression, which may explain why, in many cases, we find centers of diversity for a given crop very far from areas of diversity of the crop wild relatives. Since Harlan proposed this theory (giving as a convincing example the evolution of Helianthus species) much evidence has been found which supports it, including that cited by Dobzhansky (1973) for the speciation of Iris, Eucalyptus, Liatris, Penstemon, and Tragopogon. Introgression can thus serve as a model for what apparently happened in the case of the four Manihot diversity centers, assuming that cassava was first domesticated in one place and then carried to other places by native American peoples during their migrations, allowing extensive hybridization between cultivated species and local wild species which gave rise to numerous new species through introgression.

As was previously stated, cassava does not grow wild and it is believed that this species did not arise by natural selection, but that hybrids between some wild species may have been domesticated and maintained afterwards through vegetative reproduction. The large amount of variation in cassava cultivars due to the fact that it has been maintained for hundreds of years by vegetative reproduction makes it difficult to designate definite characters to cassava. If these 
cultivars had reproduced sexually and had been subjected to natural selection different populations with specific gene pools would have evolved depending on local environmental conditions. The hypothesis that the process of domestication included some natural hybrids and that the selected plants were maintained by vegetative reproduction for hundreds of years is supported by the fact that many experimental crosses have frequently produced hybrid cultivars between M. esculenta and local wild species (Lanjow, 1939; Nichols, 1947; Bolhuis, 1953; Jennings, 1959; Magoon et al., 1966; Abraham, 1975).

It seems that in this genus genetic and cytologic barriers are not yet well established. Support for this view comes from the work of Schmidt (1951), who states that in wild species there is a very rapid selection response in only a few generations to an increase in tuber starch content and in tuber number. It thus seems that many different wild species have the potential to increase tuber formation and starch content. M. epuinosa and M. brachyandra are two tree-species native to the Brazilian State of Bahia, which, as I have observed, are frequently grown in backyards in Goiânia, where they exhibit considerable tuber production. Migration from Bahia to Goiânia has been common during the last 30 years due to the rapid development of Goiás, and it seems that these two species have been transported by the migrants. The idea that domestication included hybrids but excluded certain wild species has been referred to by Rogers (1963) as "species complexity".

The site of domestication still needs much discussion and I prefer to use "domestication site" and not "center of origin" because it is obvious that $M$. esculenta has not arisen as a wild species by means of natural selection. Studying the history of Brazilian ethnological groups and their migration patterns can throw light on the subject, and it has been reported (Schmidt, 1951) that the Aruak people who lived in the northern Amazonia more than a thousand years ago knew of cassava and practiced agriculture, since their name in their language means "people who eat tubers". Numerous reports indicate that the Aruak cultivated cassava many centuries before Columbus, and it seems that they migrated to Central America in the 11th century, crossing the Caribbean and establishing themselves for some time in the West Indies. Many reasons have been given to explain this migration, including the need to escape from their enemies or a religious desire to find a place where "man does not die", but the most important reason is that they were probably searching for a better soil in which to cultivate cassava. Their migration coincided with the formation of a diversity center in Mexico. If cassava was carried by the Aruak to Mexico it would be expected to hybridize with local wild species, creating a new diversity center. The fact that the Aruak continued on the Bolivian Plateau and central Brazil agrees with the existence of the two diversity centers in these regions. The northeastern Brazilian diversity center is believed to be the result of migration of the Tupi-Guarani Indian group.
We must still determine which of these four centers constitutes the primary Manihot diversity center, that is the site where the genus Manihot differentiated as a biological group and from which it spread to other regions. Stebbins (1950) explains that Vavilov's concept of diversity patterns is an elaboration of the age-and-area hypothesis of Willy (Stebbins, 1950) which states that the longer a biological group occupies an area the more variable will that group be in terms of species. If we apply this concept to Manihot species, I believe that we can accept that central Brazil (a region which has long supported the growth of angiosperms) with its large number of Manihot species is the primary diversity center. This assumption finds support in the fact that species which exhibit the most primitive characteristics are restricted to this region, such species being $M$. stipularis Pax, M. pusilla Pohl and $M$. longipetiolata Pohl, which have dioecious inflorescences, and M. stricta Baillon, M. purpureo-costata Pohl and M. salicifolia Pohl, which have non-lobed and sessile leaves.

\section{RELATIONSHIPS BETWEEN MANIHOTSPECIES}

According to Rogers and Appan (1973), 98 Manihot species have been recognized, while only one species, Manihotoides pauciflora, is known in the most closely related genus, Manihotoides. Several of the attributes of M. pauciflora are not found in any Manihot species; these attributes include uniflorous inflorescences (a primitive character compared to the multi-flowered inflorescence in Manihot) and leaves born at the apex of short condensed stems arising from branchlets. Rogers and Appan (1973) classified Manihot species into 19 sections, varying from trees in the section Glazioviannae to almost acaulescent subshurbs in the section Stipularis, the species of which are also characterized by being more dioecious than monoecious, a condition reversed in all other Manihot species. Other sections, such as Tripartitue and Graciles, contain perennial subshrubs with large woody roots - their stems frequently die back to the root crown in response to drought or fires.

As has been previously stated, all Manihot species are native to tropical regions of the New World, particularly Brazil and Mexico, where Nassar (1978b) has defined four species diversity centers, i.e., Mexico and northeastern, central, and southwestern Brazil.

Manihot diversity micro-centers exist in central Brazil, where large numbers of species are concentrated in small areas less than $50 \mathrm{~km}$ in diameter (Nassar, 1978b,c; 1986), allowing frequent hybridization between species in topographically heterogeneous habitats which help to isolate fragmented gene pools leading to speciation. Tree-like species, such as M. glaziovii and M. pseudoglaziovii, are found in northeastern Brazil, while short species and subshurbs are found in central Brazil.

Wild Manihot species hybridize naturally, both with 
each other and with cassava (Nassar, 1984, 1989), and hybridization barriers within the genus appear to be weak due to the recent evolution of this group. All wild Manihot species examined cytogenetically have a high chromosome number of $2 n=36$ (Nassar, 1978a; Table II), but in spite of this the species behaves meiotically as diploids and it is believed that allopolyploidization caused the emergence of the whole group and was responsible for both its rapid speciation and the weak interspecific barriers which lead to interspecific hybridization. An extremely heterozygous gene pool is thus created, followed by differentiation, beginning a sequence of hybridization followed by speciation. Nassar (1980a) reported frequent hybridization between M. reptans Pax and M. alutacea Rogers \& Appan in sympatric natural habitats where their population boundaries overlap. Morphological marker genes for leaf color and bract size were used to identify this interspecific hybridization. The range of $M$. reptans has expanded over the last 100 years (Nassar, 1984) because the continuing gene introgression of other Manihot species has allowed $M$. reptans ecotypes to penetrate and colonize areas where unavailable pure $M$. reptans, a phenomenon also seen in other species such as $M$. cearulescens (Nassar, 1980a). From a plant-breeding viewpoint, the high value of these hybrids lies in their ability to cross with the cultigen.

Marker genes for lobe shape, presence of stem nodes, flower disc color, fruit color and fruit shape have been discovered in controlled crosses between cassava and wild Manihot species, as well as in natural hybrids between cassava and different species, and I have used these to identify hybridization. I have obtained interspecific hybrids of cassava with $M$. glaziovii, M. pseudoglaziovii, M. aesculifolia, $M$. pilosa, $M$. corymbilora, $M$. dichotoma, $M$.

Table II - Chromosome number in wild Manihot species.

\begin{tabular}{|lccc|}
\hline Species & Growth habitat & $\mathrm{N}$ & $2 \mathrm{n}$ \\
\hline M. handroana & Shrub & - & 36 \\
M. jolyana & Shrub & - & 36 \\
M. tripartita & Shrub & - & 36 \\
M. tripartita & Shrub & 18 & - \\
& & & \\
M. tweedieana & Shrub & - & 36 \\
M. humilis & Subshrub & - & 36 \\
M. pedicellaris & Shrub & - & 36 \\
M. gracilis & Subshrub & - & 36 \\
M. gracilis & Subshrub & 18 & - \\
M. dichotoma & Tree & - & 36 \\
M. giaziovii & Tree & 18 & - \\
M. giazivoii & Tree & - & 36 \\
M. anomala & & & \\
M. zehntneria & Shrub & 18 & - \\
M. olighanta & Shrub & 18 & - \\
M. nana & Subshrub & 18 & - \\
M. tomentosa & Subshrub & 18 & - \\
\hline
\end{tabular}

pohlii, M. neusana and M. anomala through controlled crosses, although their frequency was low. The meiotic behavior of several hybrids (cassava with $M$. neusana, cassava with M. pseudoglaziovii) was studied by Nassar (1992) but the results indicated low hybrid fertility between these species and cassava.

Grattapaglia et al. (1986) conducted a biosystematic analysis of wild Manihot species based on soluble seed protein patterns. Nineteen species were analyzed electrophoretically (Table III) and a species similarity matrix was constructed based on differences in band density and number (Table IV). Several species were found to be very similar, e.g., M. fruticolosa and M. pentaphylla, and $M$. pilosa and M. corymbiflora; these results correlate well with the taximetric analysis made by Rogers and Appan (1973) and show that M. pilosa and M. corymbiflora are the two species most similar to cassava. Profile analysis confirmed the introgression between $M$. cearulescens and cassava. Electrophoresis was carried out according to Laemilli (1970) using 0.1\% SDS and 5.5\% acrylamide gel in Tris- $\mathrm{HCl}(\mathrm{pH}=6.8)$, with gels being fixed for $12 \mathrm{~h}$ in $5 \%$ trichloroacetic acid followed by staining with $0.65 \%$ Coomassie brilliant blue. Four replicate gels were made for each species. The approximate molecular mass (AMM) of each band was determined according to Webber and Osborn (1969). The protein profiles varied in band intensity, and fifteen bands were selected as reference bands.

Table V shows the 15 selected reference bands (based on AMM) analyzed in each of the four replicates, with the banding classified as absent (a), weakly visible (b), visible (c), intense (d) or very intense (e). The total number of bands was calculated for each species in order to quantitatively compare the protein patterns between species. The variability of wild Manihot species in morphology, growth habit, and geographic distribution was reflected in the electrophoretic profiles as differences in the number and intensity of visible bands (Table VI).

The two cassava varieties, M. esculenta Crantz (var. $\mathrm{EAB}$ ) and M. esculenta Crantz (var. RB), shared a similarity index (SI) of $78 \%$ and were similar to some species from the Glaziovinae (54-66\% SI with M. esculenta), especially M. glaziovii Muell (66\% SI with M. esculenta) and M. pseudoglaziovii Pax \& Hoff (64\% SI with $M$. esculenta), which themselves shared an SI of $74 \%$. The Heterophyllae contained species which were most similar to the cultigen, where $M$. pilosa (67-68\% SI with $M$. esculenta) and $M$. corymbiflora (64-68\% SI with $M$. esculenta) also shared morphological similarities with cassava, indicating that they are probably part of the complex from which the cultigen originated (Nassar, 1978b). A high SI was also found between the two species of Gracilis ( $\mathrm{SI}=78 \%$ ). High similarity between species in the various sections reflects their recent speciation and is in acordance with the taxonomic classification; genetically speaking they are probably part of the same gene pool. 
Table III - Wild Manihot species and their identification number in the germplasm bank at the Universidade de Brasília.

\begin{tabular}{|c|c|c|c|c|c|c|}
\hline Species & & & Section & Habitat & No. & $\begin{array}{l}\text { No. de coleta } \\
\text { de herbário }\end{array}$ \\
\hline M. esculenta Crantz (var. EAB) & I & (A) & Manihot & Brasília (DF) & 001 & 01 \\
\hline M. esculenta Crantz (var. RB) & & (B) & Manihot & Brasília (DF) & 002 & $01 / \mathrm{a}$ \\
\hline M. zehntnieri Ule & & (C) & Heterophyllae & Goiânia (GO) & 173 & 02 \\
\hline M. grahami Hooker & II & (D) & Heterophyllae & Maringá (PR) & 375 & 03 \\
\hline M. pilosa Pohl & & (E) & Heterophyllae & São Miguel de Antes (MG) & 601 & 04 \\
\hline M. corymbioflora $\mathrm{Pax}$ & & (F) & Heterophyllae & São Miguel de Antes (MG) & 605 & 05 \\
\hline M. pohlii Wawra & & (G) & Heterophyllae & Lençóis (BA) & 139 & 06 \\
\hline M. glaziovii Muell & III & $(\mathrm{H})$ & Glaziovinae & Pentocoste (CE) & 221 & 08 \\
\hline M. pseudoglaziovii Pax 7 Hoff & & (I) & Glaziovinae & Remigio (PB) & 545 & 09 \\
\hline M epruinosa Pax \& Hoff. & & $(\mathrm{J})$ & Glaziovinae & Serra Talhada (PE) & 554 & 10 \\
\hline M. brachyandra Pax \& Hoff. & & (K) & Glaziovinae & Currais Novos (RN) & 524 & 11 \\
\hline M. reptans $\mathrm{Pax}$ & IV & (L) & Crotalariaeformes & Corumbá(GO) & 602 & 13 \\
\hline M. alutacea Rogers \& Appan & $\mathrm{V}$ & (M) & Quinquelobae & Goiás Velho (GO) & 115 & 07 \\
\hline M. fruticulosa Rogers \& Appan & VI & $(\mathrm{N})$ & Graciles & Alexânia (GO) & 162 & 10938 \\
\hline M. pentaphylla Pohl & & (O) & Graciles & Goiás Velho (GO) & 103 & 11755 \\
\hline M. stipularis $\mathrm{Pax}$ & VII & $(\mathrm{P})$ & Stipulares & Alexânia (GO) & 184 & 14 \\
\hline M. salicifolia Pohl & VIII & (Q) & Brevipetiolatae & Xavantina (MT) & 195 & - \\
\hline M. caerulescens subsp. Caerulescens & IX & (R) & Caerulescentes & Picos (PI) & 258 & 15 \\
\hline M. caerulescens (não classificada) & & (S) & Caerulescentes & Morro do Chapéu (BA) & 567 & 16 \\
\hline M. caerulescens (não classificada) & & $(\mathrm{T})$ & Caerulescentes & Jequié (BA) & 269 & 17 \\
\hline M. leptophylla $\mathrm{Pax}$ & $\mathrm{X}$ & (U) & Peruvianae & Barra do Corda (MA) & 517 & 12 \\
\hline M. neuzana Nassar & - & (V) & - & Maringá (PR) & 360 & 18 \\
\hline
\end{tabular}

Table IV - Classification of bands in wild Manihot species according to approximate molecular weight (AMW).

\begin{tabular}{|c|c|c|c|c|c|c|c|c|c|c|c|c|c|c|c|c|c|c|c|c|c|c|}
\hline \multirow{3}{*}{$\begin{array}{l}\text { AMW } \\
(\mathrm{kDa})\end{array}$} & \multicolumn{22}{|c|}{ Section } \\
\hline & \multicolumn{2}{|c|}{ I } & \multicolumn{5}{|c|}{ II } & \multicolumn{4}{|c|}{ III } & \multirow{2}{*}{$\frac{\mathrm{IV}}{\mathrm{L}}$} & \multirow{2}{*}{$\frac{\mathrm{V}}{\mathrm{M}}$} & \multicolumn{2}{|c|}{ VI } & \multirow{2}{*}{$\frac{\mathrm{VII}}{\mathrm{P}}$} & \multirow{2}{*}{$\frac{\mathrm{VIII}}{\mathrm{Q}}$} & \multicolumn{3}{|c|}{ IX } & \multirow{2}{*}{$\frac{X}{U}$} & \multirow{2}{*}{$\overline{\mathrm{V}}$} \\
\hline & A & B & C & D & E & $\mathrm{F}$ & G & $\mathrm{H}$ & I & $\mathrm{J}$ & K & & & $\mathrm{N}$ & $\mathrm{O}$ & & & $\mathrm{R}$ & S & $\mathrm{T}$ & & \\
\hline $81-75$ & - & - & - & - & - & - & - & - & - & - & - & - & - & - & - & - & - & - & - & - & - & - \\
\hline $75-66$ & 2 & 2 & 2 & 2 & 2 & 2 & 2 & 2 & 2 & 2 & 2 & 2 & 2 & 2 & 2 & 2 & 2 & 1 & 1 & 3 & 2 & 2 \\
\hline $66-62$ & 1 & 1 & 1 & 1 & 1 & 1 & 1 & - & 1 & 1 & 1 & 1 & 1 & 1 & 1 & - & 1 & - & - & 1 & - & 1 \\
\hline $62-50$ & 3 & 4 & 4 & 3 & 3 & 4 & 4 & 3 & 4 & 4 & 3 & 3 & 3 & 3 & 3 & 1 & 5 & 1 & 1 & 4 & 3 & 3 \\
\hline $50-37,5$ & 5 & 5 & 6 & 6 & 6 & 6 & 6 & 5 & 6 & 6 & 6 & 4 & 5 & 3 & 4 & 4 & 5 & 4 & 4 & 5 & 3 & 4 \\
\hline $37,5-33$ & 1 & 1 & 2 & 1 & 1 & 1 & 1 & 1 & 1 & 1 & 1 & 1 & 1 & 1 & 1 & 1 & 1 & 1 & 1 & 1 & 1 & 2 \\
\hline $33-30$ & 1 & 1 & 2 & 2 & 1 & 1 & - & 2 & 2 & - & 2 & 3 & 1 & 2 & 2 & 3 & 1 & 1 & 1 & 2 & 1 & 2 \\
\hline $30-27$ & 2 & 2 & 2 & 2 & 2 & 2 & 2 & 1 & 1 & 1 & 1 & 2 & 3 & 1 & 1 & 3 & 1 & 1 & 1 & 1 & 1 & 1 \\
\hline $27-25$ & 1 & 1 & - & - & - & - & - & - & - & - & - & - & - & - & - & - & - & - & - & 1 & - & - \\
\hline $25-24$ & - & - & 1 & - & - & - & - & - & - & - & 1 & - & - & - & - & - & - & - & - & - & - & 1 \\
\hline $24-21$ & 1 & 1 & - & - & 1 & - & - & 1 & 1 & - & 1 & 1 & 1 & 1 & 1 & 1 & 1 & - & - & - & - & 1 \\
\hline $21-20$ & - & - & 2 & 2 & - & - & 1 & 1 & - & - & - & 1 & 1 & - & 1 & - & - & - & - & 1 & - & - \\
\hline $20-18$ & 1 & 1 & 1 & 2 & - & - & 1 & - & - & 1 & - & - & 1 & - & - & - & 1 & - & - & 1 & - & - \\
\hline $18-13$ & 3 & 1 & 2 & 3 & 3 & 3 & 3 & 3 & 2 & 3 & 3 & 3 & 3 & 3 & 2 & 2 & 2 & 2 & 3 & 2 & 3 & 3 \\
\hline No. bands & 21 & 20 & 24 & 24 & 20 & 20 & 21 & 19 & 20 & 19 & 20 & 21 & 20 & 17 & 18 & 18 & 20 & 11 & 12 & 22 & 15 & 20 \\
\hline No. reference bands & 15 & 15 & 15 & 14 & 15 & 15 & 14 & 15 & 15 & 15 & 15 & 15 & 15 & 14 & 14 & 14 & 14 & 15 & 15 & 15 & 15 & 14 \\
\hline No. total bands & 36 & 35 & 39 & 38 & 35 & 35 & 35 & 34 & 35 & 34 & 36 & 36 & 35 & 31 & 32 & 32 & 34 & 26 & 27 & 37 & 30 & 34 \\
\hline
\end{tabular}

See Table III for species identification.

\section{SPECIATION WITHIN THE GENUS: THE CASE OF M. REPTANS}

In 1892, Ule found that $M$. reptans was restricted to the northern border of Minas Gerais, close to Goiás (Rogers and Appan, 1973), but I have found that it is now widespread over most of Goiás, and it appears that during the last 80 or so years this species has expanded its geo- graphical distribution and ecological range by genetic variation and interspecific hybridization. In my samples leaf shape was found to vary widely, reflecting the extent of hybridization with other Manihot species; e.g., $M$. reptans from the town of Goiás Velho was distinguished by bright red leaf veins (a characteristic of the native $M$. alutacea) and $M$. reptans had to be identified by its characteristic growth habit and its flower and inflorescence 
Table V - Distribution of reference bands according to density in studied wild Manihot species profiles.

\begin{tabular}{|c|c|c|c|c|c|c|c|c|c|c|c|c|c|c|c|c|c|c|c|c|c|c|c|}
\hline \multirow{2}{*}{\multicolumn{2}{|c|}{$\begin{array}{l}\text { Reference } \\
\text { bands } \\
\text { No. AMW }\end{array}$}} & \multicolumn{2}{|c|}{ I } & \multicolumn{5}{|c|}{ II } & \multicolumn{4}{|c|}{ III } & \multirow{2}{*}{$\frac{\mathrm{IV}}{\mathrm{L}}$} & \multirow{2}{*}{$\frac{\mathrm{V}}{\mathrm{M}}$} & \multicolumn{2}{|c|}{ VI } & \multirow{2}{*}{$\frac{\mathrm{VII}}{\mathrm{P}}$} & \multirow{2}{*}{$\frac{\mathrm{VIII}}{\mathrm{Q}}$} & \multicolumn{3}{|c|}{ IX } & \multirow{2}{*}{$\frac{X}{U}$} & \multirow{2}{*}{$\frac{=}{\mathrm{V}}$} \\
\hline & & A & B & $\mathrm{C}$ & $\mathrm{D}$ & $\mathrm{E}$ & $\mathrm{F}$ & G & $\mathrm{H}$ & I & $\mathrm{J}$ & $\mathrm{K}$ & & & $\mathrm{N}$ & $\mathrm{O}$ & & & $\mathrm{R}$ & S & $\mathrm{T}$ & & \\
\hline 1 & 81 & B & B & B & B & B & B & B & B & B & B & B & B & B & B & B & B & B & B & B & B & B & B \\
\hline 2 & 75 & B & B & B & B & B & B & B & B & B & B & B & B & B & B & B & B & B & B & B & B & B & B \\
\hline 3 & 66 & C & C & C & C & C & $\mathrm{C}$ & C & C & C & C & C & C & C & C & C & C & C & C & C & C & C & C \\
\hline 4 & 62 & $\mathrm{C}$ & $\mathrm{C}$ & $\mathrm{C}$ & $\mathrm{C}$ & $\mathrm{C}$ & $\mathrm{C}$ & $\mathrm{C}$ & $\mathrm{C}$ & $\mathrm{C}$ & $\mathrm{C}$ & $\mathrm{C}$ & $\mathrm{C}$ & $\mathrm{C}$ & $\mathrm{C}$ & $\mathrm{C}$ & $\mathrm{C}$ & $\mathrm{C}$ & $\mathrm{C}$ & $\mathrm{C}$ & $\mathrm{C}$ & $\mathrm{C}$ & $\mathrm{C}$ \\
\hline 5 & 50 & $\mathrm{C}$ & D & $\mathrm{C}$ & $\mathrm{C}$ & D & $\mathrm{C}$ & $\mathrm{C}$ & B & $\mathrm{C}$ & $\mathrm{C}$ & $\mathrm{C}$ & $\mathrm{C}$ & B & $\mathrm{C}$ & B & B & $\mathrm{C}$ & B & B & D & $\mathrm{C}$ & $\mathrm{C}$ \\
\hline 6 & 37.5 & $\mathrm{C}$ & D & $\mathrm{C}$ & $\mathrm{C}$ & $\mathrm{C}$ & $\mathrm{C}$ & $\mathrm{C}$ & $\mathrm{C}$ & $\mathrm{C}$ & $\mathrm{C}$ & $\mathrm{C}$ & $\mathrm{C}$ & B & $\mathrm{C}$ & B & B & $\mathrm{C}$ & B & B & D & $\mathrm{C}$ & $\mathrm{C}$ \\
\hline 7 & 33 & E & E & B & B & D & B & $\mathrm{C}$ & D & $\mathrm{D}$ & $\mathrm{C}$ & $\mathrm{C}$ & B & B & D & D & D & D & B & B & $\mathrm{C}$ & D & D \\
\hline 8 & 30 & $\mathrm{C}$ & B & B & $\mathrm{C}$ & $\mathrm{C}$ & $\mathrm{C}$ & $\mathrm{C}$ & $\mathrm{C}$ & $\mathrm{C}$ & E & D & $\mathrm{C}$ & B & $\mathrm{C}$ & $\mathrm{C}$ & B & C & C & $\mathrm{C}$ & $\mathrm{C}$ & $\mathrm{C}$ & $\mathrm{C}$ \\
\hline 9 & 27 & $\mathrm{C}$ & $\mathrm{C}$ & $\mathrm{C}$ & $\mathrm{C}$ & $\mathrm{C}$ & $\mathrm{C}$ & $\mathrm{C}$ & B & B & D & D & $\mathrm{C}$ & B & B & B & A & $\mathrm{C}$ & C & $\mathrm{C}$ & $\mathrm{C}$ & $\mathrm{C}$ & $\mathrm{C}$ \\
\hline 10 & 25 & B & B & B & B & B & B & $\mathrm{C}$ & B & B & B & B & $\mathrm{C}$ & B & B & C & B & C & D & D & C & B & C \\
\hline 11 & 24 & B & B & B & C & B & B & B & B & D & B & B & D & B & B & B & B & C & C & C & C & D & C \\
\hline 12 & 21 & D & D & B & B & $\mathrm{C}$ & B & C & $\mathrm{C}$ & C & $\mathrm{C}$ & $\mathrm{C}$ & $\mathrm{C}$ & B & E & $\mathrm{E}$ & E & $\mathrm{C}$ & B & B & $\mathrm{C}$ & B & $\mathrm{C}$ \\
\hline 13 & 20 & D & D & B & A & $\mathrm{C}$ & $\mathrm{C}$ & A & $\mathrm{C}$ & $\mathrm{C}$ & $\mathrm{C}$ & $\mathrm{C}$ & $\mathrm{C}$ & B & E & $\mathrm{E}$ & E & B & B & $\mathrm{C}$ & $\mathrm{C}$ & B & A \\
\hline 14 & 18 & D & D & D & C & C & D & C & D & D & D & C & C & C & B & B & B & D & B & B & D & D & B \\
\hline 15 & 13 & D & D & D & C & C & C & C & D & C & D & C & C & $\mathrm{C}$ & A & A & C & A & C & C & C & C & C \\
\hline
\end{tabular}

*See Table III for species identification. A = Absent band; B = little visible band; C = visible band; $\mathrm{D}=$ dense band; $\mathrm{E}=$ very dense band. AMW = Approximate molecular weight.

Table VI - Matrix of similarity between studied Manihot species.

\begin{tabular}{|c|c|c|c|c|c|c|c|c|c|c|c|c|c|c|c|c|c|c|c|c|c|c|}
\hline \multirow{3}{*}{$\begin{array}{l}\text { Section } \\
\text { species }\end{array}$} & \multicolumn{22}{|c|}{ Section } \\
\hline & \multirow[b]{2}{*}{ A } & \multirow[b]{2}{*}{ B } & \multicolumn{5}{|c|}{ II } & \multicolumn{4}{|c|}{ III } & \multirow{2}{*}{$\frac{\mathrm{IV}}{\mathrm{L}}$} & \multirow{2}{*}{$\frac{\mathrm{V}}{\mathrm{M}}$} & \multicolumn{2}{|c|}{ VI } & \multirow{2}{*}{$\frac{\mathrm{VII}}{\mathrm{P}}$} & \multirow{2}{*}{$\frac{\text { VIII }}{\mathrm{Q}}$} & \multicolumn{3}{|c|}{ IX } & \multirow{2}{*}{$\frac{X}{U}$} & \multirow{2}{*}{$\frac{=}{\mathrm{V}}$} \\
\hline & & & C & D & E & F & G & $\mathrm{H}$ & I & $\mathrm{J}$ & K & & & $\mathrm{N}$ & $\mathrm{O}$ & & & $\mathrm{R}$ & $S$ & $\mathrm{~T}$ & & \\
\hline A & - & 78 & 54 & 45 & 67 & 64 & 58 & 66 & 64 & 58 & 58 & 58 & 50 & 45 & 43 & 43 & 54 & 30 & 32 & 54 & 47 & 50 \\
\hline B & & - & 49 & 38 & 68 & 68 & 68 & 61 & 60 & 56 & 54 & 50 & 52 & 42 & 41 & 44 & 52 & 28 & 31 & 53 & 43 & 50 \\
\hline $\mathrm{C}$ & & & - & 62 & 51 & 65 & 48 & 51 & 49 & 51 & 54 & 54 & 59 & 31 & 30 & 32 & 45 & 33 & 33 & 50 & 44 & 40 \\
\hline D & & & & - & 47 & 53 & 65 & 40 & 45 & 40 & 50 & 54 & 47 & 30 & 29 & 30 & 39 & 32 & 33 & 50 & 39 & 59 \\
\hline E & & & & & - & 75 & 61 & 70 & 75 & 63 & 74 & 66 & 62 & 46 & 44 & 45 & 60 & 36 & 39 & 66 & 53 & 62 \\
\hline $\mathrm{F}$ & & & & & & - & 58 & 67 & 71 & 67 & 70 & 70 & 71 & 42 & 40 & 41 & 58 & 36 & 38 & 58 & 56 & 56 \\
\hline G & & & & & & & - & 51 & 54 & 51 & 65 & 65 & 52 & 38 & 39 & 38 & 50 & 34 & 36 & 50 & 41 & 78 \\
\hline $\mathrm{H}$ & & & & & & & & - & 74 & 71 & 60 & 55 & 61 & 49 & 50 & 48 & 56 & 35 & 37 & 64 & 54 & 52 \\
\hline I & & & & & & & & & - & 59 & 64 & 70 & 45 & 45 & 43 & 43 & 62 & 41 & 45 & 70 & 69 & 60 \\
\hline $\mathrm{J}$ & & & & & & & & & & - & 74 & 55 & 52 & 43 & 41 & 42 & 52 & 45 & 44 & 36 & 49 & 48 \\
\hline K & & & & & & & & & & & - & 71 & 59 & 41 & 39 & 38 & 52 & 32 & 34 & 37 & 47 & 54 \\
\hline $\mathrm{L}$ & & & & & & & & & & & & - & 59 & 38 & 39 & 46 & 56 & 33 & 36 & 38 & 51 & 59 \\
\hline M & & & & & & & & & & & & & - & 40 & 43 & 50 & 50 & 32 & 35 & 37 & 47 & 50 \\
\hline $\mathrm{N}$ & & & & & & & & & & & & & & - & 78 & 55 & 50 & - & 88 & 38 & 43 & 42 \\
\hline $\mathrm{O}$ & & & & & & & & & & & & & & & - & - & 51 & & - & 39 & 37 & 43 \\
\hline $\mathrm{P}$ & & & & & & & & & & & & & & & & & 36 & & & - & 38 & 41 \\
\hline Q & & & & & & & & & & & & & & & & & - & & & & 49 & 56 \\
\hline $\mathrm{R}$ & & & & & & & & & & & & & & & & & & & & & 49 & 36 \\
\hline $\mathrm{S}$ & & & & & & & & & & & & & & & & & & & & & 50 & 38 \\
\hline $\mathrm{T}$ & & & & & & & & & & & & & & & & & & & & & & \\
\hline U & & & & & & & & & & & & & & & & & & & & & 43 & 58 \\
\hline
\end{tabular}

See Table III for species identification.

morphology. Genes donated from different species adapted to different environments have allowed this species to rapidly expand over the whole State of Goiás. Harlan (1961) gives the example of Helianthus annuus (the annual sunflower) which has acquired a vast gene pool due to the formation of hybrids with at least six other Helianthus species.

\section{HYBRIDIZATION OF CASSAVA AND ITS WILD RELATIVES}

Two wild Manihot species, M. neusana Nassar and M. anomala Pax (maintained in a living collection at the Experimental Biology Station of the University of Brasília), were used for the creation of interspecific hybrids 
with cassava by controlled crosses employing vector insects (Nassar, 1989).

In October 1982 the wild species were each planted in three rows alternating with cassava. In June 1983, 200 seeds were collected from each species and replanted in October 1984 for identification of possible natural hybridization. Marker genes for variegated fruit color (smooth = dominant), red flower-disk (yellow = dominant), setaceous bracteole (foliaceous $=$ dominant) and noded stem (smooth = dominant) were used to identify interspecific hybrids. Growth habit, height, stem texture, and tuber formation were also recorded. In addition to the open pollination, 400 manual crosses with pollen of cassava cultivar Catelo were realized. Only 43 seedlings grew from the $200 \mathrm{M}$. neusana seeds collected, of which only 2 hybrids were identified. Interspecific hybrids were identified by dominant markers from cassava: noded stem, setaceous bracteoles, ribbed fruit, and tuberculated root (Table VII), along with other characters which provided indirect evidence of hybridization. The 200 seeds from M. anomala gave rise to 112 seedlings, of which 3 seedlings showed signs of interspecific hybridization. Only one seedling survived to maturity, and this hybrid exhibited dominant phenotypes from cassava, i.e., ribbed fruit, red flower-disk, noded stem, and tuberous roots (Table VII). These results show that glabrous stem, setaceous-foliaceous bracteoles, red/creamy flowerdisk, variegated-green fruit, and ribbed/unribbed fruit are simple marker genes that can be used to recognize interspecific hybridization. It is evident that interspecific barriers between Manihot species can be broken by the use of an abundant diversity of pollinator gametes transmitted by insect vectors, although in these and other earlier crosses, interspecific crosses were difficult to fertilize manually (Nassar, 1980a). The evidence suggests that barriers between cassava and other Manihot species are weak and recently evolved, and it seems they have arisen not as a primary isolating event but secondarily after geographic isolation. Nassar (1978b) postulated that cassava itself is an interspecific hybrid that appeared by domestication some 2000 years ago or less.

\section{INTERSPECIFIC MANIHOTHYBRIDS}

The wild Manihot species M. neusana Nassar has been hybridized with the cassava clone Catelo through controlled hybridization with the help of pollinating insects (Nassar, 1989) to obtain an interspecific hybrid that combined marker genes (ribbed fruit from cassava and variegated fruit color from M. neusana) from both parents. This hybrid (HN) was backcrossed with cassava and used as a pollinator in one trial and as a fruit carrier in another trial. Seeds were obtained from both crosses, but only one plant could be raised from each cross. HO1 resulted when the interspecific hybrid (HN) was the maternal plant (seed carrier), and $\mathrm{HO} 4$ resulted from crosses where the interspecific hybrid (HN) was used as pollinator. The meiotic and mitotic behaviors of the three hybrid plants ( $\mathrm{HN}, \mathrm{Hl}$ and $\mathrm{H} 4$ ) were cytogenetically studied. For the study of meiosis, inflorescences were fixed in 3:1 absolute alcohol:glacial acetic acid and refrigerated for $24 \mathrm{~h}$ before staining the anthers with aceto-carmine. Metaphase chromosome configurations, chromosome distribution in anaphase I and tetrad formation were also studied. Pollen viability was determined using the aceto-carmine/iodine stain (Nassar, 1978a). For mitotic studies, root tips were left in $0.2 \%$ colchicine for $2 \mathrm{~h}$, fixed in acetic-alcohol for $24 \mathrm{~h}$, treated with $1 \mathrm{~N} \mathrm{HCl}$ for $10 \mathrm{~min}$ and then stained with aceto-carmine.

\section{MEIOTIC BEHAVIOR OF FI HYBRIDS (HN)}

For the interspecific hybrid between $M$. neusana and cassava, 100 pollen metaphase I mother cells (PMCs), 30 metaphase II PMCs and 1000 tetrads were studied. The metaphase I PMCs showed different chromosome configurations (Table VIII), with a high average bivalent frequency

Table VII - Comparison of morphological characters of M. neusana, cassava and their hybrid.

\begin{tabular}{|lccc|}
\hline Character & M. neusana & Cassava & Hybrid \\
\hline Growth habit & Procumbent shrub & Erect shrub & Erect shrub \\
& $1.5-2 \mathrm{~m}$ & $1.5-2 \mathrm{~m}$ & $1.5-2 \mathrm{~m}$ \\
Youn stem texture & Hairy & Glabrous & Hairy \\
Bracteoles & Foliaceous & Setaceous & Setaceous \\
Fruits & Globose, without & Ovoid, & Ovoid, ribbed, \\
& ribs, variegated & ribbed, green & variegated \\
Tuber formation & None & Forms tubers & Forms tubers \\
Growth habit & Erect shrub & Erect shrub & Erect shrub \\
& $2-2.5 \mathrm{~m}$ & $1.5-2 \mathrm{~m}$ & $1.5-2 \mathrm{~m}$ \\
Youn stem texture & Hairy & Glabrous & Hairy \\
Bracteoles & Semi-foliaceous & Setaceous & Setaceous \\
Flower disk color & Creamy & Red & Red \\
Leaf form & Anomala & Lobed; 5 lobes & Anomala \\
Fruit & Globose, without ribs & Ovoid ribbed & Ovoid ribbed \\
Tuber formation & Scarcely forms tubers & Forms tubers & Forms tubers \\
\hline
\end{tabular}


of $16.13 \%$, probably attributable to a lack of synapses between chromosomes or to failure of the two species to remain associated. Virtually the only other report on this subjects is that of Magoon et al. (1966), who studied chromosome pairing in the interspecific hybrid resulting from the cross between $M$. glaziovii and cassava and found regular synapsis, which led them to conclude that there is a strong relationship between the two species. Nassar et al. (1986) have suggested that the M. glaziovii used by Magoon et al. was not pure-bred M. glaziovii but rather a natural interspecific hybrid between $M$. glaziovii and cassava. If this is true then the supposed interspecific hybrid would in fact be a backcross. In our studies, the study of anaphase I has shown that 38 out of 40 PMCs exhibited laggards, attributable to the occurrence of univalents resulting from nonhomologous chromosomes.

Anaphase II showed meiotic restitution, and 5 of the 33 PMCs studied in this phase exhibited a second meiotic restitution (SMR), forming 36 chromosomes at each pole. Apparently this phenomenon is a consequence of meiotic disturbance in the hybrid, an example which was the breakdown of anaphase I, probably due to disharmony between the two different genomes. Nassar (1991) previously documented this phenomenon in interspecific hybrids between cassava and M. pseudoglaziovii. The presence of such restitution was confirmed in the following tetrad stage, where the formation of both dyads and tetrads was observed.

The partial fertility of the backcrossed generation (HO1) shows that the species M. neusana may be classified within the secondary gene pool of cassava according to the concept of Harlan and de Wet (1980). Other Manihot species that may fit in this category are: M. melanobasis (Jennings, 1959), M. glaziovii (Magoon et al., 1966), M. reptans, $M$. zenhtneri, $M$. anomala, $M$. oligantha, $M$. pohlii, M. dichotoma, M. epruinosa, and M. leptophylla (Nassar et al., 1986). It was concluded that the cassava hybrid with $M$. neusana showed irregular meiotic behavior in the lack of complete chromosome pairing, formation of univalents in metaphase I, chromosome retardation in anaphase I, micronuclei in the tetrad stage and meiotic restitution.

\section{APOMIXIS AND ITS ROLE IN MANIHOTSPECIATION}

Apomixis was found to occur frequently in Manihot species, which may explain why there is rapid speciation in this genus because polyploidy may offer the heterozygosity necessary for initial speciation and any hybrids produced can be maintained through apomixis. To verify the presence of apomixis and study its anatomic nature in cassava, Nassar et al. (1997, 1998 et seq.) selected two putative apomictic cassava clones (number 031 and 200) which had been selected, based on vigor, from the $\mathrm{F}_{1}$ population of a cross between an interspecific hybrid (M. dichotoma $\mathrm{x} M$. esculenta) and the cultivated clone "Branca Santa Catarina". Progenies from both clones were studied for embryo-sac structure and molecular behavior.

\section{Embryo-sac analysis}

Morpho-structural development of embryo sacs was studied histologically. Unpollinated pistillate buds (presumably 1-day pre-anthesis) and pollinated pistils at postanthesis were collected in the field (7.30-12.00 a.m.) and immediately fixed in Farmer's fixative (1:3, glacial acetic acid:95\% ethanol) for subsequent dissection under a dissection microscope (X40, transmitted light). Dissected ovules were dehydrated in an ethanol series and cleared overnight in benzyl-benzoate-four-and-a half $\left(\mathrm{BB}-4_{1 / 2}\right)$ fluid (2:2:2:2:1:1(w/w), lactic acid:chloral hydrate:phenol: clove oil:xylene:benzyl benzoate; Herr Jr., 1982) as previously reported by Ogburia and Adachi (1994). One hundred and twenty-seven ovules from clone 031 and one hundred and thirty-four ovules of clone 200 were used in the embryo-sac analysis. Transparent ovules ( 127 of clone 031 and 134 of clone 200) were observed using an Olympus BX50 microscope equipped with Nomarski's differential interference contrast (DIC) optics and a 100-W high pressure mercury lamp with appropriate filters for viewing and photography. Both megasporangia and megagametophytes were photographed and subsequently printed using a Sony color video printer (Mavigraph UP-1200).

\section{DNA extraction and RAPD assay}

Total genomic DNA was isolated from $200 \mathrm{mg}$ of fresh leaf tissue ground in liquid nitrogen using the CTAB protocol of Doyle and Doyle (1987), modified by the addition of 1\% PVP and 1\% 2-mercaptoethanol to the isolation buffer. DNA concentration was estimated by gel electrophoresis comparing the fluorescence intensities of the ethidium bromide-stained samples to those of lambda DNA standards.

For the RAPD assay, working stocks of genomic DNA were diluted in water at a concentration of $2.5 \mathrm{ng} / \mu \mathrm{l}$. Arbitrary ten-base primers (kits OP-A through OP-Z) were obtained from Operon Technologies Inc. (Alameda, CA, USA). Amplification reactions $(13 \mu \mathrm{l})$ were carried out according to Williams et al. (1990) with the following modifications: $0.4 \mathrm{mM}$ of ten-base primer, $10 \mu \mathrm{g} / \mu \mathrm{l}$ non-acetylated BSA (New England Biolabs), 5 to $10 \mathrm{ng}$ of genomic DNA and 1 unit of Taq DNA-polymerase. Amplifications were

Table VIII - Metaphase I chromosome configurations of interspecific Manihot hybrids and their parents.

\begin{tabular}{lcccc|} 
& PMC (N) & Trivalents & Mean bivalents & Univalents \\
\hline N. neusana & 20 & - & 18.00 & - \\
Cassava & 20 & - & 18.00 & - \\
GN & 100 & - & 17.00 & 1.58 \\
HO1 & 30 & 1.86 & 16.13 & 0.13 \\
HO4 & 100 & 1.63 & 12.41 & 8.84 \\
\hline
\end{tabular}

$\mathrm{PMC}=$ Pollen mother cells. 
performed in 96-well microwell plates using an MJ Research PT-100 thermal controller. RAPD products were analyzed by electrophoresis in $1.5 \%$ or $2.0 \%$ agarose gels containing $0.2 \mathrm{mg} / \mathrm{ml}$ ethidium bromide. Gel images were captured and digitalized with an Eagle-Eye 11 system (Stratagene, CA, USA).

Gel scoring was performed directly from the gel images on a computer screen and images stored electronically on a laser CD. A set of 24 arbitrary primers previously selected for high multiplex content and discrimination power in cassava (Grattapaglia et al., 1996) were used, and the presence or absence of RAPD fragments was scored by visual inspection of the gel images. Informative RAPD markers were identified as described previously (Grattapaglia and Sederoff, 1994).

Two replicate RAPD analysis experiments including DNA extractions, RAPD assays and marker scoring were carried out with the set of select primers on the putatively apomictic individuals to confirm the observed band patterns. Two of the maternal parents and 67 offspring were genotyped with 24 selected arbitrary ten-base primers. Each selected primer amplified an average of 8.25 clearly interpretable RAPD fragments with a range of 5 to 14 fragments. A total of 198 clearly interpretable and reproducible RAPD markers were surveyed in this study, a number that was considered to provide representative genome coverage for the objective of this study.

Progenies of clones 031 and 200 displayed highly uniform DNA fingerprints, but, except for one individual in each progeny set, it was possible to find markers that readily showed that individuals were not derived from apomixis. In the progeny of clone 031 , individual 4 showed a pattern of RAPD bands identical to that of individual 5 in the maternal progeny and an identical pattern was also observed in individual 5 of the progeny of clone 200. Although it may seem unlikely that the maternal parent and one of its zygotic progeny could have an identical combination of over 100 RAPD fragments, it is possible. Using the statistical procedure described by Novy et al. (1994), Grattapaglia et al. (1996) showed that the correspondence between parent and progeny is not, in this case, an artifact resulting from a limited number of RAPD markers surveyed but rather has a biological basis. Given the number of markers surveyed, the probability that complete uniformity in RAPD markers between the maternal parent and their respective progeny individuals happened due to chance alone was equal or less than $10^{-3}$ in both clones. Putative apomixis was therefore detected in progenies of both clones 031 and 200, at a rate of 3.13 and 2.70, respectively. These results clearly indicate that the type of apomixis detected in this study is facultative and occurs at a very low frequency in cassava.

A total of 261 ovules were analyzed histologically and in both clones aposporic sacs were found inside the sexual embryo sacs; the presence of 2 embryo sacs in an ovule indicates the aposporitory nature of apomixis. It seems that one embryo sac is derived from somatic ovule cells, while the second sac is derived from a normal megaspore mother cell. At a certain stage before the complete maturation of the sexual embryo sac, it may abort and be replaced by an aposporous sac or it may continue to develope and give rise to two embryo sacs and, consequently, two embryos in the ovule. This abnormality was verified in $2.36 \%$ and $1.49 \%$ of the ovules of clones 031 and 200, respectively. Similar results using the same histological clarification technique were reported in Cenchrus ciliaris (Young et al., 1979). With the help of DIC microscopy, it was possible to see in the cleared pistils details of the cassava embryo sac. The normal sacs showed an egg, two polar nuclei, three antipodals and, occasionally, synergids, with the egg often being inconspicuous. The antipodals were distinguished by their swollen tear-drop shape, dense cytoplasm, chalazal position, and the absence of a wall separating them from the sac cavity. The aposporous sacs lacked antipodals and had only one nuclei per sac. Sometimes there was a single polar nucleus and an egg. These observations strongly suggest that apospory (development of aposporic embryo sacs) is the mechanism responsible for apomixis in cassava - apospory being the most common mechanism responsible for apomixis in angiosperms. In this type of apomixis the aposporic embryo sac originates from one or more of the ovule somatic cells, followed by sac enlargement and vacuolation (Asker, 1980). This type of apomixis explains the multiple plants per seed found in clone 031 by Nassar $(1994 a, b)$. The presence of apomixis in clones 031 and 200 shows the potential that wild species have as a source of genes for apomixis in cassava, since clone 031 represents the $\mathrm{F}_{2}$ generation of a cross between cassava and M. dichotoma, while clone 200 is the $\mathrm{F}_{1}$ hybrid of cassava and $M$. glaziovii. Genes controlling apomixis in wild species related to corn, sugar beet, wheat, and several forage grasses have already been reported (Asker, 1979), while Nassar et al. (1998) have provided further molecular and embryonic evidence for the occurrence of apomixis in two putative apomictic clones of cassava.

\section{THE ROLE OF UNREDUCED GAMETES POLYPLOIDIZATION}

Polyploids were found to be produced by unreduced gamete fertilization (Nassar, 1992), and it seems that the formation of unreduced microspores (gametes with somatic chromosome number) appears to be a common phenomenon in angiosperms (Harlan and de Wet, 1975; Wet, 1980), where they most likely have a major role in the evolution and origin of polyploids. From a plant breeding point of view these gametes are important since they may lead to the development of highly productive triploids and tetraploids by sexual reproduction, and may also be important for preserving heterozygosity (Mendiburu and Peloquin, 1977).

The most convincing cytological evidence for the occurrence of unreduced gametes in higher plants has probably come from research on microspores, such as that car- 
ried out by Prakken and Swaminathan (1952) who observed that dyads (with two reduced microspores) and tetrads (with four reduced microspores) both occur in the same plant in several species of Solanum, observations later confirmed in other crop plants (Roades and Dempsey, 1966).

Nassar (1992) first reported that unreduced microspores occur in interspecific hybrids of cassava as a consequence of meiotic irregularity, and now there is general agreement that dyads form due to spindle abnormalities which may be visible at meiotic metaphases I and II (Nassar et al., 1996). Cassava clones grown in the germplasm bank of the Centro Agronomico Tropical de Investigacion y Ensenanza, CATIE (Costa Rica), were used to show the presence of unreduced microspores in cassava (Vasquez and Nassar, 1994). Floral buds were collected and fixed for $24 \mathrm{~h}$ in 3:1 ethanol-acetic acid, transferred to $70 \%$ ethanol and stored at $5^{\circ} \mathrm{C}$. Anthers were squashed in a drop of $1 \%$ acetocarmine, and metaphase I chromosomes counted and chromosomal associations noted. The tetrad stage was observed to determine the frequency of dyads and triads, with about 300 tetrads being counted in each clone. Photomicrographs were taken from temporary preparations using a Zeiss standard research microscope.

Out of the nine cassava clones studied, eight showed regular metaphase with complete pairing and the formation of 18 bivalents. The ninth clone (No. 6477, popularly known as Chioriqui) showed a sectorial chimera in the inflorescence. One sector of inflorescence developed flowers with a normal metaphase I and complete pairing of chromosomes as well as no laggards at anaphase I and no micronuclei at the tetrad stage, while the other sector developed flowers with an extremely abnormal metaphases I (lack of chromosome pairing asynapsis) as well as empty anthers. In the 20 metaphases examined the chromosome association ranged from 11 to 12 bivalents instead of 18 normal bivalents, with the remaining chromosomes forming univalents only, resulting in the formation of 1-15 micronuclei per tetrad (Tables IX, X). This chimeral sector was apparently due to a gene mutation in the second layer (LII) of the apex of the growing shoot. Since only a lateral sector showed this abnormality, the chimera must have been sectorial rather than mericlinal or periclinal.

This is the first report of a mutation which affects chromosome pairing in cassava, and since cassava reproduces vegetatively, it is likely that this mutation has been preserved for a long time in this indigenous Costa Rican clone. To confirm OK unreduced microspore formation, dyads and triads were checked in 300 tetrads of each clone but only clone 11965 produced dyads (11 dyads per 300 sporocytes), indicating first meiotic restitution (Table XI). Triads were formed by clones 11965,9959 (Mangi), 6429, Vegna Mochera, and 6399 at a frequency of 1 to $1.3 \%$.

There is variation in $2 \mathrm{n}$ gamete production among cassava clones (Table X), and several workers have reported that in species with a tendency to form unreduced microspores the frequency of such gametes may vary from one line to another while stable high $2 \mathrm{n}$ gamete production has been observed in $2 \mathrm{n}$ gamete producer lines (Mok and Peloquin, 1975). The variation in the frequency of $2 \mathrm{n}$ gametes may be attributed mainly to genotypic differences and it seems that unreduced microspore formation is gene controlled and not due to the disturbance of chromosome asynapsis. It has, however, been supposed that unreduced microspore formation is due to the occurrence of nonfunctional spindles, resulting in all the metaphase chromosomes remaining in the center instead of separating to the poles (Vorsa and Bingham, 1979).

The causes of unreduced microspores in plants are variable, ranging from simple recessive genes (Mok and Peloquin, 1975) to disturbed spindle function in interspecific hybrids. Nassar et al. (1995) suggested that the disturbance of meiotic division in interspecific hybrids may lead to a higher frequency of aneuploid gametes, making it possible to select polyploids from among their progeny. Brazilian Manihot species are believed to be the progenitors of cassava, and Brazil contains several cassava diversity centers (Nassar, 1978b). Interspecific hybridizations have been systematically carried out by my group since 1980, and a large number of interspecific hybrids representing a vast array of variation have been produced (Nassar, 1989). Some of these hybrids (or their progenies) OK were used in the work reported in this paper because they presented large genetic variation; they were: $\mathrm{F}_{1} M$. glaziovii $\mathrm{x}$ cassava (three genotypes), $\mathrm{F}_{2} M$. epruinosa $\mathrm{x}$ cassava, $\mathrm{F}_{2} M$. anomala $\mathrm{x}$ cassava (three genotypes), $\mathrm{F}_{3}$ M. pseudoglaziovii x cassava (two genotypes) and $\mathrm{F}_{4}$ M. pseudoglaziovii $\mathrm{x}$ cassava.

Table IX - Chromosome association, diad, and triad frequencies in different cassava clones.

\begin{tabular}{|c|c|c|c|c|c|}
\hline \multirow[t]{2}{*}{ Cultivar } & \multirow{2}{*}{$\begin{array}{l}\text { Chromosome } \\
\text { association }\end{array}$} & \multicolumn{2}{|l|}{ Diads } & \multicolumn{2}{|c|}{ Triads } \\
\hline & & Frequency & $2 \%$ & Frequency & $2 \%$ \\
\hline 9959 (Mangi) & $18_{\text {II }}$ & - & - & 4 & 1.33 \\
\hline 6417 (White) & $18_{\text {II }}$ & - & - & - & - \\
\hline 10861 (Num-4-RB) & $18_{\text {II }}$ & - & - & - & - \\
\hline 6429 (Negra Muchera) & $18_{\text {II }}$ & - & - & 4 & - \\
\hline 11965 (Sim Nombre) & $18_{\text {II }}$ & 11 & 3.7 & 3 & 1 \\
\hline 6379 (Amarilla-1) & $18_{\text {II }}$ & - & - & 3 & 1 \\
\hline 6473 (Vagana 4208) & $18_{\text {II }}$ & - & - & - & - \\
\hline \multicolumn{6}{|l|}{6477 (Chioriqui) } \\
\hline Chimera a & $18_{\text {II }}$ & & & & \\
\hline Chimera b & $11_{\mathrm{II}}+14_{\mathrm{I}}$ & & & & \\
\hline
\end{tabular}

Table X - Frequency of diads and five kinds of tetrads in the sectorial chimera of clone 6477 (No. of tetrads studied $=300$ ).

\begin{tabular}{|llcrrrr|}
\hline Parameter & Diad & $\begin{array}{c}\text { Normal } \\
\text { tetrads }\end{array}$ & \multicolumn{3}{c|}{$\begin{array}{c}\text { Tetrads with different } \\
\text { numbers of micronuclei }\end{array}$} \\
\hline Frequency & 1 & 18 & 26 & 41 & 26 & 188 \\
Percent & 0.3 & 6 & 8.7 & 13.9 & 8.9 & 62.6 \\
\hline
\end{tabular}


Table XI - Frequency and percentage of diads and triads in different sporads of Manihot hybrids.

\begin{tabular}{|c|c|c|c|c|c|c|c|c|c|c|c|c|}
\hline & \multirow{2}{*}{$\begin{array}{l}\text { Microspores } \\
\text { examined }\end{array}$} & \multicolumn{2}{|c|}{ Diad } & \multicolumn{2}{|c|}{ Triad } & \multicolumn{2}{|c|}{ Tetrad } & \multicolumn{2}{|c|}{ Abnormal tetrad } & \multirow{2}{*}{$\begin{array}{c}\text { Pollen } \\
\text { examined }\end{array}$} & \multicolumn{2}{|c|}{ Pollen viability } \\
\hline & & $\mathrm{n}$ & $\%$ & $\mathrm{n}$ & $\%$ & $\mathrm{n}$ & $\%$ & $\mathrm{n}$ & $\%$ & & $\mathrm{n}$ & $\%$ \\
\hline M. glaziovii x cassava & 1217 & 10 & 0.82 & 8 & 0.66 & 1158 & 95.15 & 41 & 3.37 & 2979 & 1197 & 40.18 \\
\hline $\begin{array}{l}F_{1} M . \text { glaziovii } x \text { cassava } x \\
\text { cassava (1st genotype) }\end{array}$ & 1168 & 43 & 3.70 & 8 & 0.70 & 1107 & 94.80 & 10 & 0.80 & 1713 & 723 & 42.21 \\
\hline $\begin{array}{l}\mathrm{F}_{1} M . \text { glaziovii } \mathrm{x} \text { cassava } \mathrm{x} \\
\text { cassava (2nd genotype) }\end{array}$ & 1044 & 0 & 0.00 & 4 & 0.40 & 1040 & 99.60 & 0 & 0.00 & 1463 & 1123 & 76.76 \\
\hline M. epriinosa $\mathrm{x}$ cassava & 1252 & 0 & 0.00 & 0 & 0.00 & 1252 & 100.00 & 0 & 0.00 & 603 & 567 & 94.03 \\
\hline $\begin{array}{l}\mathrm{F}_{2} M . \text { anomala } \mathrm{x} \text { cassava } \\
(1 \text { st genotype })\end{array}$ & 1374 & 1 & 0.07 & 0 & 0.00 & 1326 & 96.51 & 47 & 3.42 & 836 & 183 & 21.89 \\
\hline $\begin{array}{l}\mathrm{F}_{2} M . \text { anomala } \mathrm{x} \text { cassava } \\
\text { (2nd genotype) }\end{array}$ & 1145 & 0 & 0.00 & 0 & 0.00 & 1117 & 95.56 & 28 & 2.44 & 1297 & 441 & 34.00 \\
\hline $\begin{array}{l}\mathrm{F}_{2} M . \text { anomala } \mathrm{x} \text { cassava } \\
\text { (3rd genotype) }\end{array}$ & 1136 & 1 & 0.09 & 0 & 0.00 & 1106 & 97.36 & 29 & 2.55 & 801 & 452 & 56.43 \\
\hline $\begin{array}{l}\mathrm{F}_{3} M . \text { pseudoglaziovi } \mathrm{x} \\
\text { cassava }\end{array}$ & 1416 & 0 & 0.00 & 0 & 0.00 & 1134 & 95.62 & 62 & 4.38 & 1427 & 873 & 61.18 \\
\hline $\begin{array}{l}\mathrm{F}_{4} M . \text { pseudoglaciovi } \mathrm{x} \\
\text { cassava }\end{array}$ & 1210 & 0 & 0.00 & 0 & 0.00 & 1207 & 98.80 & 3 & 0.20 & 1130 & 1034 & 94.50 \\
\hline $\begin{array}{l}\mathrm{F}_{1} M . \text { dichotoma } \mathrm{x} \text { cassava } \\
\text { (1st genotype) }\end{array}$ & 1138 & 1 & 0.09 & 0 & 0.00 & 1125 & 98.80 & 12 & 1.05 & 1704 & 862 & 50.59 \\
\hline $\begin{array}{l}\mathrm{F}_{1} M . \text { dichotoma } \mathrm{x} \text { cassava } \\
\text { (2nd genotype) }\end{array}$ & 491 & 2 & 0.40 & 3 & 0.60 & 476 & 98.86 & 10 & 2.04 & 1273 & 299 & 23.49 \\
\hline
\end{tabular}

Pollen viability, meiotic metaphase I chromosome associations, and the occurrence of dyads and triads have been studied in the above hybrids. For the meiotic studies inflorescences were fixed in a 3:1 mixture of absolute alcohol:glacial acetic acid and kept at $5^{\circ} \mathrm{C}$ for $24 \mathrm{~h}$, after which the anthers were stained with acetocarmine and metaphase I chromosome configurations and pair-formation were observed. For pollen viability studies, 1-3 flowers per plant were selected and their pollen crushed in acetocarmine, after which pollen counts were made and the percentage of stained normal pollen were calculated.

Chromosome associations and their frequency in meiotic metaphase I PMCs of all interspecific hybrids have shown regular pairing of the 18 bivalents except for the progeny of $M$. glaziovii x cassava (first genotype), which revealed the presence of two univalents in $5 \%$ of the cells examined. This was the same genotype that showed a high frequency $(3.7 \%)$ of dyad formation and low pollen viability $(42 \%)$. The study of PMCs in the tetrad phase revealed the formation of abnormal tetrads having 1-3 micronuclei in all hybrids used in the experiment, except $\mathrm{F}_{1} M$. glaziovii $\mathrm{x}$ cassava (2nd genotype). The abnormal tetrads ranged from $0.8 \%$ in $\mathrm{F}_{1}$ M. glaziovii x cassava (1st genotype) to $94 \%$ in the $\mathrm{F}_{1}$ hybrid M. epruinosa $\mathrm{x}$ cassava (Table XI).

The high frequency of unreduced microspores in the $\mathrm{F}_{2}$ progeny of $M$. glaziovii x cassava will facilitate the use of this genotype and its progeny as a possible progenitor of polyploids in the future. Dyad formation is apparently due to meiotic restitution, while the low pollen viability may be due to univalent formation and the consequent irregular chromosome distribution leading to unbalanced gametes. Judging by its chromosome number and the com- plete pairing of its meiotic metaphase chromosomes, cassava is a natural allopolyploid (Nassar, 1978b; Vasquez and Nassar, 1994). If unreduced gametes were responsible for its natural polyploidization in the past it is possible that one can detect them among wild relatives and their hybrids with cassava, and, as has been mentioned before, the presence of unreduced microspores in the hybrid progeny confirms this hypothesis.

Vasquez and Nassar (1994) have reported a high frequency of unreduced microspores among cassava clones and it is believed that this character is heritable and genetically controlled, probably having been acquired by cassava from its wild ancestors (Nassar et al., 1995) and thus representing an evolutionary remnant. This is significant because it provides direct evidence of polyploidization from lower ploidy levels by the mechanism of unreduced gametes and not other types of somatic doubling (Harlan and de Wet, 1975; de Wet, 1980). Accordingly the genes of the wild ancestors of cassava have probably had the opportunity to combine and produce the larger rooted cassava that we now know (Nassar, 1992; Nassar et al., 1996), and it seems that this character is correlated with meiotic irregularities if there is univalent formation in the genotype producing unreduced microspores.

It is worth mentioning that this unreduced microspore-producing genotype is a progeny of a natural hybrid of M. glaziovii with cassava which has been maintained by farmers for hundreds of years through vegetative reproduction, and which probably arose through recurrent mutation and has been maintained through vegetative reproduction. In germplasm that reproduces sexually, it would have been eliminated by natural selection due to the abortion of the gametes that carried this trait. 


\section{ACKNOWLEDGMENTS}

The collection of live wild Manihot species was established at the University of Brasília with the help of the International Development Research Center (IDRC), Ottawa, to which this author is grateful.

\section{REFERENCES}

Abraham, A. (1975). Breeding of tuber crops in India. Indian J. Genet. Plant Breed. 17: 212-217.

Asker, S. (1979). Progress in apomixis research. Hereditas 91: 231-240.

Asker, S. (1980). Gametophytic apomixis: elements and genetic regulation. Hereditas 93: 277-293.

Bolhuis, G.G. (1953). A survey of some attempts to breed cassava varieties with a high content of proteins in the roots. Euphytica 20: 107-112.

de Wet, J.M.J. (1980). Origin of polyploids. In: Polyploidy: Biological Relevance (Lewis, W.H., ed.). Plenum Press, New York.

Dobzhansky, T. (1973). Genética do Processo Evolutivo. Tradução de Celso Abbade Mourão. Polígono Ed., São Paulo.

Doyle, J.J. and Doyle, J.L. (1987). Isolation of plant DNA from fresh tissues. Focus 12: 13-15.

Grattapaglia, D. and Sederoff, R.R. (1994). Genetic linkage maps of Eucalyptus grandis and E. uroplylla using a pseudo-testcross strategy and RAPD markers. Genetics 137: 1121-1137.

Grattapaglia, D.E., Nassar, N.M.A. and Dianese, J. (1986). Biossistemática de espécies brasileiras do Gênero Manihot baseada em padrões de proteína da semente. Ciênc. Cult. (Journal of the Brazilian Association for the Advancement of Science) 19: 294-300.

Grattapaglia, D., Costa e Silva, C. and Nassar, N.M.A. (1996). Strict maternal inheritance of RAPD fingerprints confirms apomixis in cassava (Manihot esculenta Crantz). Can. J. Plant Sci. 76: 379-382.

Harlan, J. (1961). Geographic origin of plants useful to agriculture. Am. Assoc. Adv. Sci. 66: 3-9.

Harlan, J.R. and de Wet, J.M.J. (1975). On o Wings and a prayer; the origin of polyploids. Bot. Rev. 41:361-390.

Herr Jr., H.M. (1982). An analysis of methods for permanently mounting ovules cleared in four and a half type clearing fluids. Stain Technol. 57: $161-165$

Jennings, D.L. (1959). Manihot melanobasis Muell. Arg. - a useful parent for cassava breeding. Euphytica 8: 157-162.

Laemilli, U.K. (1970). Cleavage of structural proteins during the assembly of the head of bacteriophage T4. Nature 227: 680-685.

Lanjow, J. (1939). Two interesting species of Manihot L. from Surinam. Recl. Trav. Bot. Neerl. 36: 542-549.

Magoon, M.L., Jos, J.S. and Appan, S.G. (1966). Cytomorphology of Interspecific Hybrid Between Cassava and Ceara Rubber. Chromosome Information Service, Japan, 8-10.

Mendiburu, A.O. and Peloquin, S.J. (1977). The significance of 2n gametes in potato breeding. Theor. Appl. Genet. 49: 53-61.

Mok, D.W.K. and Peloquin, S.S. (1975). The inheritance of three mechanisms of diploandroid ( $2 \mathrm{n}$ pollen) formation in diploid potatoes. Heredity 35: 295-302.

Nassar, N.M.A. (1978a). Genetic resources of cassava: Chromosome behavior in some Manihot species. Indian J. Genet. Plant Breed. 38: 135137.

Nassar, N.M.A. (1978b). Conservation of the genetic resources of cassava (Manihot esculenta Crantz). Determination of wild species localities with emphasis on probable origin. Econ. Bot. 32: 311-320.

Nassar, N.M.A. (1978c). Microcenters of wild cassava Manihot spp. Diversity in Central Brazil. Turrialba 28: 345-347.

Nassar, N.M.A. (1980a). Attempts to hybridize wild Manihot species with cassava. Econ. Bot. 34: 13-15.

Nassar, N.M.A. (1980b). The need for germplasm conservation in wild cassava. Indian J. Genet. Plant Breed. 39: 465-470.

Nassar, N.M.A. (1984). Natural hybrids between Manihot reptans Pax and M. alutaced Rogers \& Appan. Can. J. Plant Sci. 64: 423-425.

Nassar, N.M.A. (1986). Genetic variation of wild Manihot species native to Brazil and its potential for cassava improvement. Field Crops Res. 13: 177-184.
Nassar, N.M.A. (1989). Broadening the genetic base of cassava, Manihot esculenta Crantz, by interspecific hybridization. Can. J. Plant Sci. 69: 1071-1073.

Nassar, N.M.A. (1991). Production of triploid cassava, Manihot esculenta Crantz by hybrid diploid gametes. Field Crops Res. 13: 173-182.

Nassar, N.M.A. (1992). Cassava in South America: A plant breeder view. Ciênc. Cult. (Journal of the Brazilian Association for the Advancement of Science) 44: 25-27.

Nassar, N.M.A. (1994a). Selection and development of apomitic cassava clone. Ciênc. Cult. (Journal of the Brazilian Association of the Advancement of Science). 41:168-171.

Nassar, N.M.A. (1994b). Relationship between Manihot species: A review proceeding of the 2nd Internacional Scientific Meeting of the Cassava Biotechnology Network, Bogor, 90-100.

Nassar N.M.A. (1995). Development of cassava interespecif hybrids for savanna (cerrado) conditions. J. Root Crops 22: 9-17.

Nassar, N.M.A. and O'Hair, S.K. (1985). Variation among clones in relation to seed germination. Indian J. Genet. Plant Breed. 45: 429-432.

Nassar, N.M.A., Silva, J.R. and Vieira, C. (1986). Hibridação interespecífica entre mandioca e espécies silvestres do Manihot. Ciênc. Cult. (Journal of the Brazilian Association for the Advancement of Science) 33: 1050-1055.

Nassar, N.M.A., Nassar, H.N.M., Vieira, C. and Saraiva, S.L. (1995). Cytogenetic behavior of interespecific hybrids of cassava and M. neusansa Nassar. Can. J. Plant Sci. 75: 675-678.

Nassar, N.M.A., Nassar, H.N.M., Carvalho, C.G. and Vieira, C. (1996). Induction of a productive aneuploid in cassava, Manihot esculenta Crantz. Braz. J. Genet. 19: 123-125.

Nassar, N.M.A., Nassar, H.N.M. and Freitas, M. (1997). Prospects of polyploidizing cassava, Manihot esculenta Crantz, by unreduced microspores. Plant Breed. 116: 195-197.

Nassar N.M.A., Vieira, M.A., Vieira, C. and Grattapaglia, D. (1998). Molecular and embryonic evidence of apomix in cassava interespecific hybrids (Manihot spp.). Can J. Plant Sci. 78: 348-352.

Nichols, R.F.W. (1947). Breeding cassava for resistance. Est. Afr. Agric. J. 12: 184-194.

Novy, R.G., Kobak, C., Gofreda, J. and Vorsa, N. (1994). RAPDs identify varietal misclassification and regional divergence in cranberry (Vaccinium macrocarpon Ait.) Pursh. Theor. Appl. Genet. 88: 1004-2020.

Ogburia, M.N. and Adashi, T. (1994). An improved cleared pistil technique for rapid in toto observation of embryo sac malformation in cassava Manihot esculenta Crantz. Proceedings of the 2nd International Scientific Meeting in Cassava Biotechnology Network, Indonesia, pp. $117-127$.

Prakken, R. and Swaminathan, M.S. (1952). Cytological behaviour of some interspecific hybrids in the genus Solanum. Genetics 54: 505-522.

Roades, M.M. and Dempsey, E. (1966). Induction of chromosome doubling at meiosis by elongated gene in maize. Genetics $54: 505-522$.

Rogers, D.J. (1963). Studies on Manihot esculenta Crantz and related species. Bull. Torrey Bot. Club 99: 43-54.

Rogers, D. and Appan, C. (1973). Manihot, Manihotoides, Euphorbiaceae. Flora Neotropica Hafner Press, New York, NY.

Schmidt, C.B. (1951). A mandioca, contribuição para o conhecimento de sua origem. Bol. Agric. No. 1:1 (São Paulo).

Stebbins, G.L. (1950). Variation and Evolution in Plants. Columbia Press, New York.

Vavilov, N.I. (1951). Phytogeographic basis of plant breeding. The origin, variation, immunity and breeding of cultivated plants. Chron. Bot. 13: 1-366.

Vasquez, N. and Nassar, N.M.A. (1994). Unreduced microspores in cassava, Manihot esculenta Crantz clones. Ind. J. Genet. 54: 436-441.

Vorsa, N. and Bingham, E.T. (1979). Cytology of pollen formation in diploid alfalfa, Medicago sativa. Can. J. Genet. Cytol. 21: 526-530.

Webber, K. and Osborn, M.J. (1969). The reliability of molecular weight determination by sulfate polyacrylamide gel electrophoresis. J. Biol. Chem. 244: 4406-4412.

Williams, J.G.K., Kubelik, A.R., Livak, K.J., Rafalski, J.A. and Tingey, S.V. (1990). DNA polymorphisms amplified by arbitrary primers are useful as genetic markers. Nuclei Acids Res. 18: 6531-6535.

Young, B.A., Sherwood, R.T. and Bashaw, E.C. (1979). Cleared-pistil and thick sectioning techniques for detecting aposporous apomixis in grasses. Can. J. Bot. 57: 1668-1672. 